\title{
Marquer un temps, avant d'aboutir : les manuscrits de Wang Wen-hsing
}

\section{Peng Yi}

Traducteur : Christine Collière

\section{OpenEdition \\ Journals}

Édition électronique

URL : http://journals.openedition.org/genesis/1320

DOI : $10.4000 /$ genesis. 1320

ISSN : 2268-1590

\section{Éditeur :}

Presses universitaires de Paris Sorbonne (PUPS), Société internationale de génétique artistique littéraire et scientifique (SIGALES)

\section{Édition imprimée}

Date de publication : 15 avril 2014

Pagination : 165-180

ISBN : 9782840509370

ISSN : $1167-5101$

Référence électronique

Peng Yi, « Marquer un temps, avant d'aboutir : les manuscrits de Wang Wen-hsing », Genesis [En ligne], 38 | 2014, mis en ligne le 27 juin 2016, consulté le 12 juin 2020. URL : http://journals.openedition.org/ genesis/1320; DOI : https://doi.org/10.4000/genesis.1320 


\section{Marquer un temps, avant d'aboutir: les manuscrits de Wang Wen-hsing}

Peng Yi

\section{En guise de commencement}

À bien des égards, cet article n'est qu'une étude préalable à une introduction plus complète aux manuscrits chinois ou à l'œuvre de Wang Wen-hsing (écrivain taïwanais né en 1939)1. En effet, un tel travail demanderait non seulement que l'on parcoure un champ historique bien plus vaste que celui que nous prétendons couvrir, d'autant qu'il ne manque pas d'études générales sur le sujet, mais aussi que l'on étudie ce champ d'une manière qui permette de faire émerger une perspective sur les modes d'écriture et de travail de la génération de Wang. Comprendre les manuscrits de Wang est un travail exigeant, pour lequel une solide connaissance de la tradition dans laquelle il s'inscrit est indispensable et la présente étude ne pourra donc être qu'une ouverture, proche des balbutiements et des frémissements des origines.

Directement ou indirectement, le destin de Wang et des écrivains de sa génération a précisément été fait de commencements ou de recommencements qu'ils ont dû surmonter. La défaite du gouvernement nationaliste chinois en 1949, qui eut pour conséquence un exode massif vers Taïwan, ne fut pas la cause directe de l'émigration de la famille de Wang, qui quitte dès 1946 la province côtière du Fujian, de l'autre côté du détroit de Taïwan, pour s'installer dans l'île. Cependant, les changements cataclysmiques de l'autre côté du détroit allaient être doublement ressentis par Wang et les écrivains expatriés comme lui sur une île qui venait tout juste de mettre fin à un demi-siècle de relations coloniales avec le Japon. On peut présenter les choses ainsi : la réinvention du langage et de la forme, ou leur importation (notamment en provenance du symbolisme, du surréalisme et du modernisme, le plus souvent par le biais de traductions) a été nécessaire pour suturer les plaies et combler les pertes. En ce sens, et en particulier pour un auteur comme Wang qui attache une grande valeur à la langue traditionnelle, commencer à écrire signifie inévitablement un combat contre la nécessité d'employer une voix d'emprunt, que l'on n'a pas librement choisie, si tant est qu'on vous en ait donné une2. D'autre part, les multiples sens que revêt le commencement nous rappellent l'importance d'examiner les brouillons de l'écrivain, afin de pouvoir par la suite mieux comprendre ce qu'implique, formellement et historiquement, l'acte de commencer.

1. Les manuscrits examinés ici sont tirés des deux romans publiés de Wang Wen-hsing, Jiabian (Processus familial) et Beihai de ren (titre provisoire de la traduction française à venir chez Allia : Un homme dos à la mer), et d'une œuvre en cours. Le premier a été d'abord publié en feuilleton dans six numéros du Chungwai Literary Monthly de septembre 1972 à février 1973, puis publié par Huan yu en 1973, un an après sa publication en feuilleton. La version la plus répandue est celle publiée par Hong fan en 1978 et c'est celle à laquelle nous faisons référence dans le présent article. Le deuxième roman, Beihai de ren, est composé de deux volumes, publiés successivement par Hong fan en 1981 puis en 1999. L'œuvre en cours, d'après l'auteur, traite d'abord et avant tout de thèmes religieux. Le reste de l'œuvre de Wang est constitué de recueils de nouvelles, de textes en prose et de conférences.

2. La relation du romancier à la tradition littéraire chinoise ne se limite pas à une éducation traditionnelle sur laquelle un enfant n'a pas de contrôle. L'intérêt de Wang est non seulement attesté par les fréquentes et insistantes déclarations de l'auteur lui-même, mais également par des publications récentes de chercheurs de plus en plus conscients de l'importance de cette relation. Un exemple typique en est Endless War: Fiction, Essays by Wang Wen-hsing, important recueil d'articles dans lequel Shu-ning Sciban, le coordonnateur du volume, met ce lien particulièrement en évidence. 
Notre but ici est de proposer une brève description de manuscrits de Wang Wen-hsing. La description de ces documents nous amène à une étude préliminaire des processus de travail de Wang et en particulier à la dernière étape de la genèse, c'est-à-dire le passage du brouillon à la mise au net. Comme nous le verrons dans la troisième partie, qui s'appuie sur les matériaux plus complets provenant de l'œuvre en cours et sur la description par l'auteur de ses habitudes de travail, l'étape la plus difficile du processus de composition est celle qui consiste à copier le brouillon pour le mettre au net. Deux particularités supplémentaires viennent caractériser ce processus et constituent une possible occasion de l'aborder sous l'angle génétique. D'une part, le brouillon est quasi identique à la mise au net pour ce qui est des éléments « substantifs » (au sens où l'entend la critique textuelle ${ }^{3}$ ), les différences apparaissant principalement dans les éléments « accidentels », c'est-à-dire la ponctuation et l'orthographe. D'autre part, la langue - qui est l'une des caractéristiques les plus difficiles et les plus controversées du deuxième roman de Wang, dans la mesure où le récit est inextricablement absorbé par le monologue du personnage principal - est fabriquée morceau par morceau (littéralement, comme nous le verrons). C'est seulement au moment qui précède immédiatement l'avènement de la mise au net (qui est écrite en clair, sur des feuilles de papier réglé pour le typographe, voir fig. 1), que le véritable défi de l'écriture et de la mise en forme est surmonté. C'est là le temps de suspension avant d'aboutir auquel le titre de cet essai fait référence, et j'espère, en me concentrant sur ce point dans ma dernière partie, pouvoir suffisamment justifier que l'on s'arrête sur son importance génétique et extra-génétique.

3. N.D.T. : Ces termes sont courants dans la critique textuelle anglosaxonne depuis le célèbre article « The Rationale of Copy-Text » de W.W. Greg. Voir Peter Shillingsburg et Paul Eggert, « Le paysage éditorial anglo-américain de 1980 à $2005 »$ (trad. C. Collière), Genesis, n 30, « Théorie : état des lieux », 2010, p. 35-39.

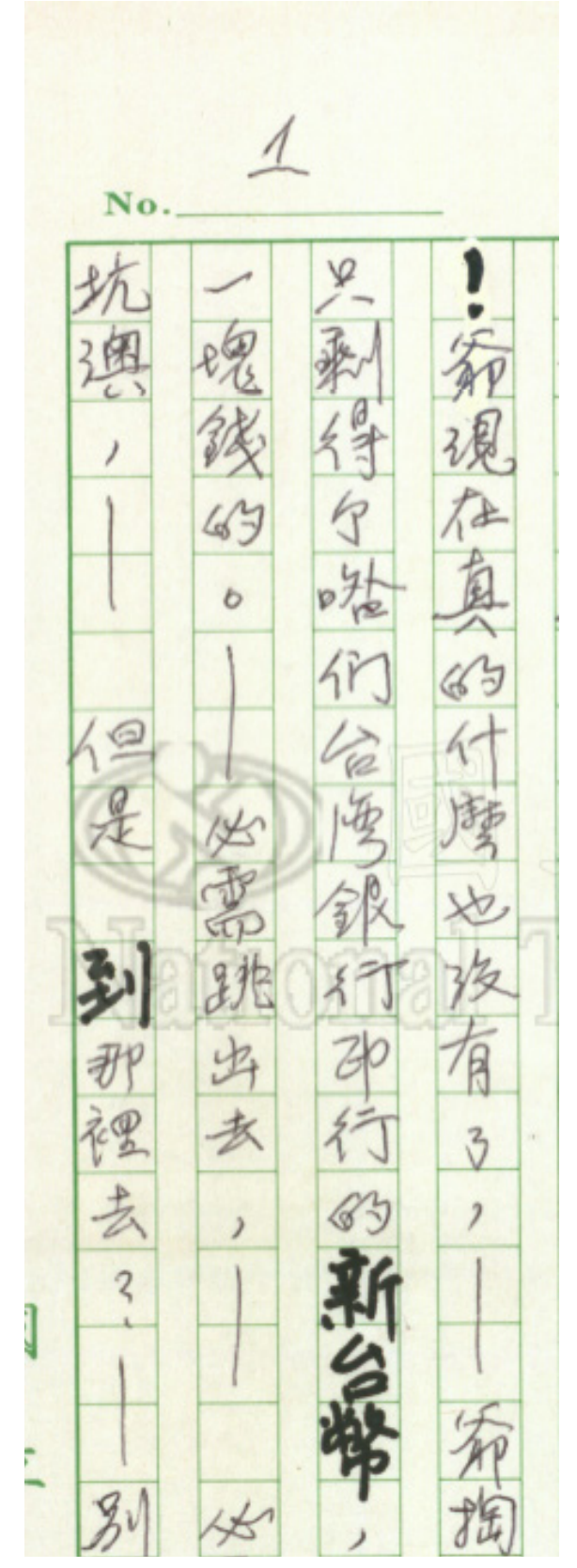

Fig. 1 : Wang Wen-hsing, mise au net de Beihai de ren, $\mathrm{f}^{\circ} 1$ (détail) (National Taiwan University Library, Special Collections, MF0001-05-001) 


\section{Wang Wen-hsing et ses brouillons}

Un simple coup d'œil, même rapide, aux brouillons de Wang Wen-hsing suffit à soulever des questions. Le brouillon de Jiabian (Processus familial, 1973), le premier roman majeur de l'écrivain moderniste 4 , est déjà surchargé d'un protosystème de signes qui exigent une explication, voire un véritable décryptage. Ces signes sont pour l'essentiel des variations sur le système de ponctuation moderne standard, avec quelques innovations significatives. La langue dans laquelle le roman est écrit, comme son sujet (la dissolution d'une famille, présentée comme inévitable, ce qui trouble et glace une société qui, dans les périodes de crises majeures, se raccroche désespérément à cette valeur, fondamentale pour elle), ont provoqué une controverse dont les effets se font encore sentir. Les figures 2 et 3 montrent les expérimentations sur la typographie et la ponctuation qui abondent dans les brouillons et dans la version publiée. Ainsi, un large éventail de ponctuations expérimentales est visible dans le détail de la figure 2, qui correspond à trois lignes complètes, l'auteur utilisant des feuilles blanches de format $\mathrm{A} 4$ et rédigeant de gauche à droite, horizontalement 5 . À la première ligne, on observe que l'auteur entoure de cercles (indiqués ici par un triangle noir) les mots écrits à l'aide des sinogrammes simplifiés de Chine continentale, qui sont utilisés parfois à Taïwan par commodité ou en privé, afin de se souvenir de les remplacer par les sinogrammes traditionnels correspondants utilisés à Taïwan. On observe également à la même ligne un tiret (indiqué ici par un rectangle horizontal), et nous avons signalé par un ovale l'endroit où Wang a entouré le symbole de transcription phonétique

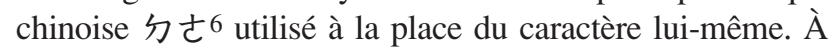
la deuxième ligne, on voit une virgule horizontale (dans un rectangle horizontal), un punctus au-dessus du caractère (dans l'ovale central) et une autre virgule horizontale, tracée d'un trait plus épais (dans le triangle). À la troisième ligne, qui est la dernière du folio, on observe tout un enchaînement de signes, commençant par un signe de pause (dùnhào), indiqué par le rectangle vertical, signe courant dans la ponctuation traditionnelle chinoise, appelée $j u$ dou, mais qui n'appartient pas au système de ponctuation moderne. Dans l'ovale au bout de cette ligne, à droite, trois signes différents se détachent : le punctus et les deux barres obliques qui sont modulables et qui définissent à la fois l'intonation et la durée du mot. Dans ces expérimentations, on trouve des signes inventés à partir de signes de ponctuation existants, comme les virgules horizontales en gras, ou la réactivation de signes chinois traditionnels ju dou, comme le signe de pause. Ces symboles expérimentaux sont fonction de la situation et ont pour but d'assurer la précision de l'intensité, c'est-à-dire du phrasé. En ce sens, ils se situent entre l'improvisation et le système, c'est-à-dire qu'ils relèvent d'un protosystème.

Les figures 2 et 3 témoignent du caractère ajustable des marques : dans le carré de la figure 3, on rencontre même une barre oblique de staccato. Il faut préciser que si nous savons que la barre oblique de la figure 2 est ajustable, c'est d'une part parce que le romancier a laissé sur le brouillon des indications de régie très claires qui précisent pour le typographe les différentes longueurs des barres obliques de façon à ce qu'elles

4. Le qualificatif d' « écrivain moderniste » est bien sûr discutable, du moins à cause de la nature même de la relation dialectique du modernisme à la tradition, et surtout à cause des périodisations politiquement chargées de l'historiographie de la littérature taïwanaise. Par commodité, j'ai adopté le terme le plus couramment utilisé.

5. Traditionnellement, on écrit en partant du bord droit de la page blanche, comme on le voit sur la plupart des exemples de calligraphie. Le fait que Wang parte du bord opposé, c'est-à-dire qu'il écrive de gauche à droite, n'implique cependant pas une rupture radicalement significative par rapport à la pratique habituelle. En effet, la direction traditionnelle existe toujours, mais la prévalence de la coutume occidentale est également omniprésente. Si le sens d'écriture choisi par Wang dans ses manuscrits reflète l'influence occidentale prédominante, les feuilles réglées qu'il utilise pour préparer les mises au net (comme dans la figure 1) suivent l'ancienne coutume et sont conçues de telle sorte que les mots soient généralement écrits verticalement, du haut vers le bas et de droite à gauche. Ce format est souvent utilisé pour donner un statut formel ou officiel au texte.

6. Ce que l'on appelle le système de transcription phonétique chinoise, ou zhu yin fu hao, fut inventé au début du $\mathrm{Xx}^{\mathrm{e}}$ siècle dans le but de faciliter la prononciation universelle standardisée qui remplaça la méthode pédagogique traditionnelle. Ces symboles, combinés aux quatre tons, apparaissent généralement à côté des caractères dans les manuels. Ce système est encore utilisé à Taïwan. Il est différent du pinyin, qui est un système consistant essentiellement à romaniser les mots et qui a officiellement remplacé le système zhu yin en 1958 en République populaire de Chine. Surtout, le pinyin est indissociablement lié à toutes sortes de questions qui définissent en grande partie les voies de la modernisation empruntées par la Chine. Voir Jing Tzu, Sound and Script in Chinese Diaspora, Cambridge, Mass., Harvard University Press, 2010. 


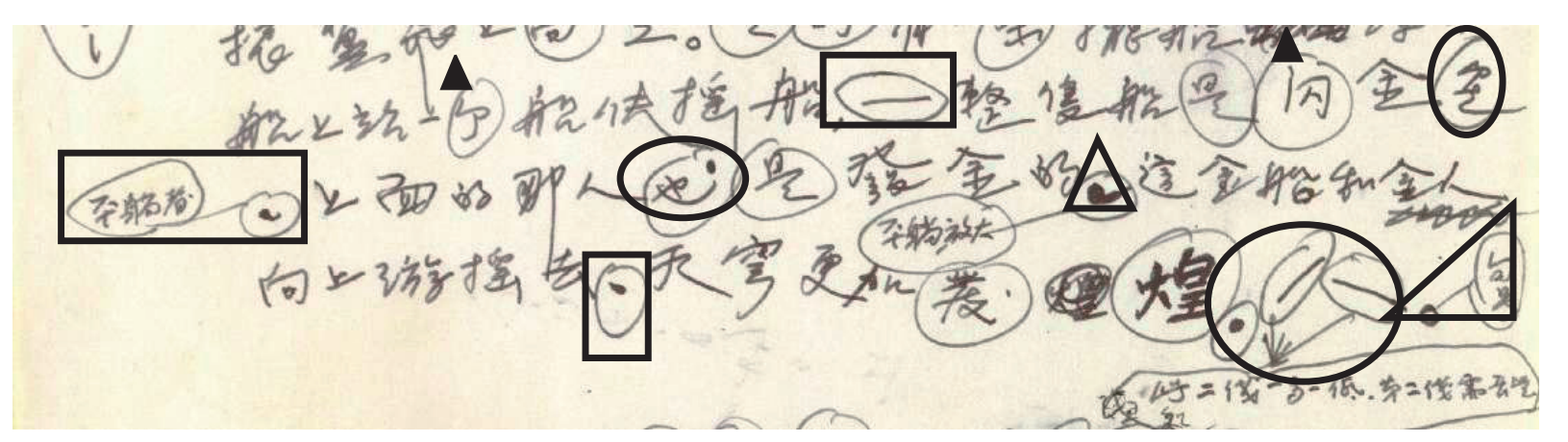

Fig. 2 : Wang Wen-hsing, manuscrit de Jiabian, $\mathrm{f}^{\circ} 102 \mathrm{r}^{\circ}$ (détail)

(National Taiwan University Library, Special Collections, MF0001-01-105)

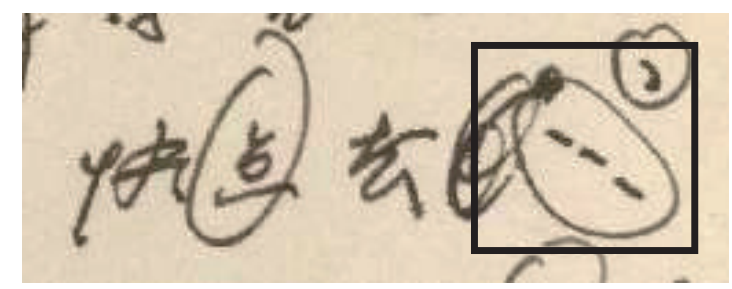

Fig. 3 : Wang Wen-hsing, manuscrit de Jiabian, $\mathrm{f}^{\circ} 148 \mathrm{r}^{\circ}$ (détail) (MF0001-01-151)

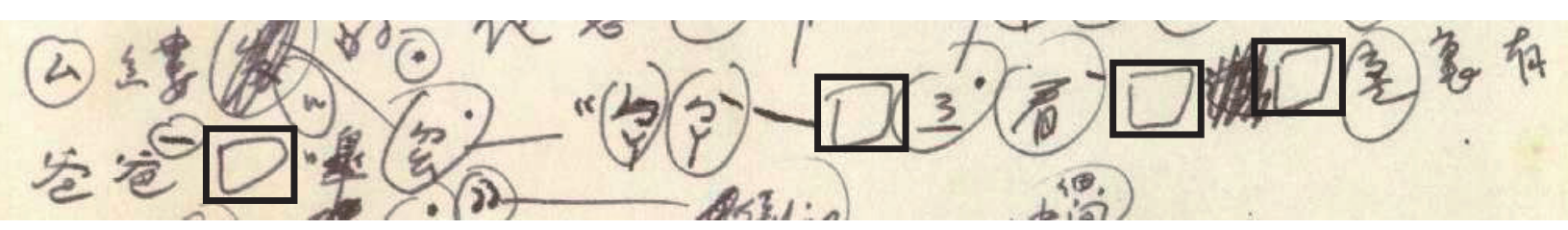

Fig. 4 : Wang Wen-hsing, manuscrit de Jiabian, $\mathrm{f}^{\circ} 117 \mathrm{r}^{\circ}$ (détail) (MF0001-01-120) 
correspondent à la durée voulue, d'autre part parce que le punctus plein après le dernier caractère de la phrase n'en indique pas la fin grammaticale : c'est le cercle vide final, selon les indications de l'auteur, qui remplit cette fonction. Le punctus plein, le cercle vide et les instructions autographes sont indiqués par le triangle en bas à droite de la figure 2. Les barres obliques ne peuvent que décrire la vie de la phrase au-delà de ses limites grammaticales légitimes : ce sont peut-être les échos et la voix de la scène tout entière, portés encore et encore par son rythme, c'est-à-dire par ce qui se fonde sur la phrase, voire sur le texte même, mais qui les dépasse.

L'ensemble des figures 2, 3 et 4 comprend les caractéristiques principales des brouillons de l'auteur. Du début à la fin de Jiabian, puis dans les deux volumes de Beihai de ren (Un homme dos à la mer), son deuxième roman, l'emploi des symboles phonétiques se poursuit. En revanche, les signes de ponctuation du premier roman, qui, ensemble, constituent presque à eux seuls une sorte de paratexte, ne sont pas tous réutilisés dans le deuxième. La presque totalité des signes de ponctuation de Wang sont des commentaires sur le texte auquel ils appartiennent, et bien que les éléments « accidentels » soient porteurs de significations « substantives », ce sens est étroitement lié aux divers contextes dans lesquels ils se trouvent dans le texte ou le brouillon. Cette immanence caractérise la nature apparemment paratextuelle de cette ponctuation et sa complexité situationnelle : parce qu'ils appartiennent en propre aux chantiers en cours, ces signes ne sont pas suffisamment isolés pour devenir un paratexte de plein droit, et par conséquent, en raison de leur nature de protosystème $a d$ hoc, ils sont sujets à variation, à la fois empiriquement et théoriquement ${ }^{7}$. Ces signes de ponctuation se situent entre un paratexte à proprement parler et un commentaire en aparté sur la composition en cours et pourraient être considérés comme faisant partie intégrante du processus génétique.

Cette variabilité affecte l'écriture de Wang, et l'examen du brouillon, dans lequel le travail d'écriture et les objectifs de l'auteur apparaissent de façon convaincante, va en définir notre compréhension. Le manuscrit que nous étudions ici nous a fourni des exemples remarquables en ce qu'ils représentent à la fois des tendances typiques et le rêve d'un Texte idéal dont le texte existant tente de s'approcher. En effet, peut-être pour des raisons pratiques, la version publiée de Jiabian ne conserve qu'une partie de ces signes. Lorsqu'on en arrive à son roman plus tardif, Beihai de ren, les systèmes de notation qui donnent ses caractéristiques distinctives au manuscrit du premier roman se sont unifiés et simplifiés, ce qui clarifie le brouillon aussi bien que la version publiée.

Dans la figure 4, également extraite du premier roman, nous trouvons déjà une particularité qui dominera le roman suivant : la mobilisation de l'espace vide. Les quatre zones indiquées par des carrés semblent être des pauses arrachées à la continuité de la syntaxe. Du moins, le premier espace suivant les deux premiers caractères remplit cette fonction. Le deuxième, après les deux symboles phonétiques et la barre oblique inversée, remplit une fonction supplémentaire : étant située après une barre oblique inversée décrivant la longueur du phrasé, cette zone vide ne fait pas partie du contour prosodique, par conséquent marque peut-être un temps, un silence, qui est une négation de la prosodie. Pourtant, les troisième et quatrième carrés parviennent à représenter le double de la quantité de temps et de silence. En multipliant par deux le silence, les carrés semblent en faire un objet, une chose. Mais comment mesure-t-on le silence, et si on le mesure précisément, comment peut-on le multiplier ou le diviser de façon exacte ? Wang n'a pas eu recours, ou s'est interdit d'avoir recours aux outils plus systématiques de la notation musicale, comme les points d'orgue ou

7. Je fais ici allusion à la distinction que pose Genette entre les «paratextes », tels qu'il les définit dans Seuils, et les «pré-textes », ou avanttextes, catégorie à laquelle appartiennent les brouillons : " "Voici ce que l'auteur a consenti à nous laisser savoir de la façon dont il a écrit ce livre." De "laisser savoir" à faire savoir, il n'y a qu'un pas fort léger, et du coup le de jure réinvestit en force le de facto : pour visiter une "fabrique", il faut bien que la fabrique existe, et que quelqu'un l'ait ouverte » (Seuils, Paris, Éditions du Seuil, 1987, p. 364). Tous les points soulevés ici sont d'importance : la question de l'intention, celle de la rhétorique de l'authenticité des matériaux génétiques et celle du statut scientifique du paratexte comme objet définissable par opposition à l'avant-texte. Dans le cas des brouillons étudiés ici, le protosystème de ponctuation tend vers une logique de la lecture, mais s'il fonctionnait, il ne serait pas l'œuvre littéraire ou le manuscrit qu'il est, mais autre chose, un projet de réforme linguistique ou un langage artificiel, par exemple. 


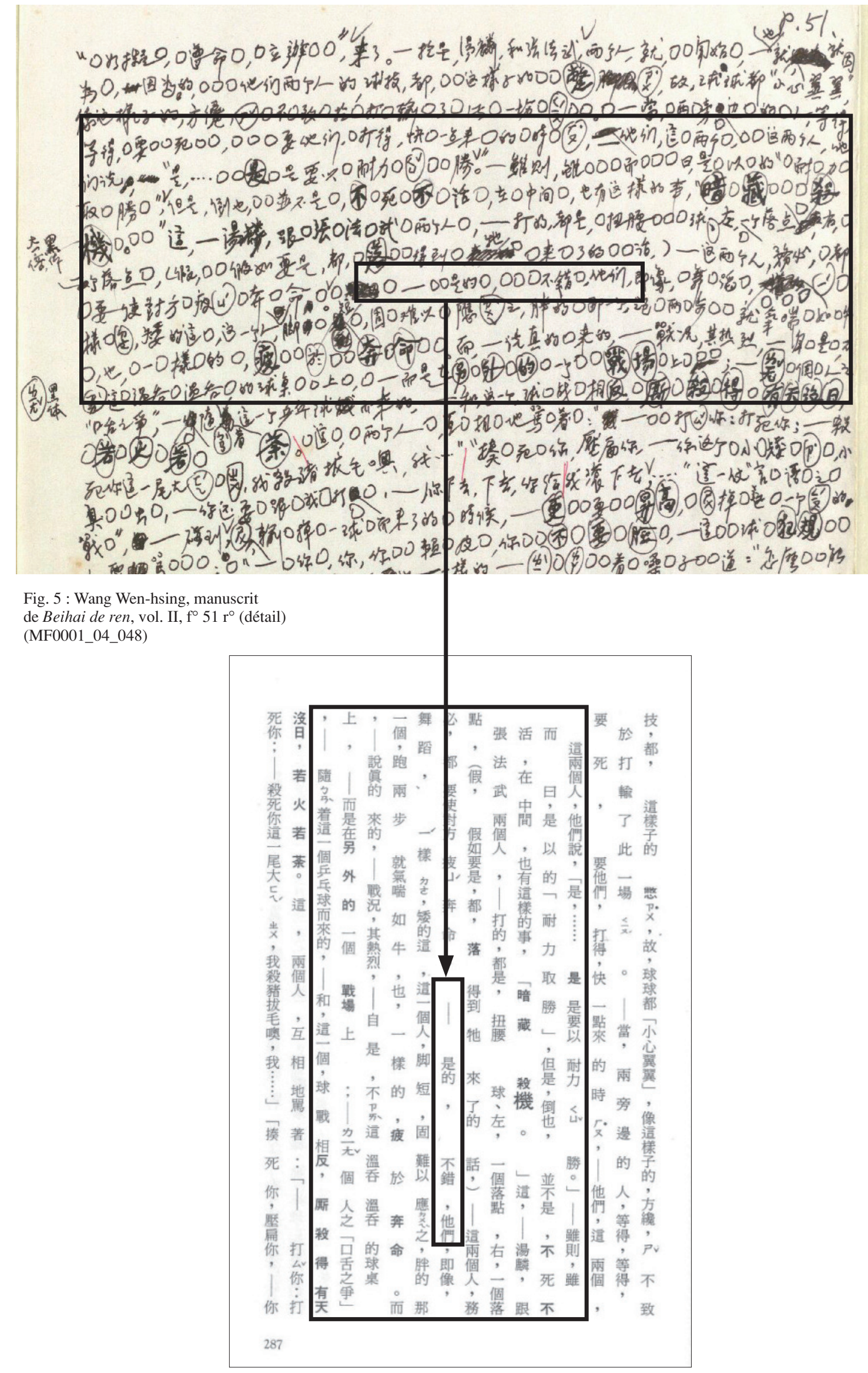


les césures, ou aux moyens plus textuels de Beckett ${ }^{8}$, justement à cause de la nature semi-systématique et non textuelle inhérente aux carrés-silences, nature qui affectera à la fois la genèse et la forme ultime du texte dans le roman suivant.

Lorsqu'on se tourne vers le manuscrit du deuxième roman, Beihai de ren, on voit (fig. 5) que l'auteur a éliminé la complexité débordante du premier roman et que le foisonnement des signes de ponctuation s'est réduit à une utilisation sélective des symboles phonétiques. Dans les rectangles des figures 5 et 6 , reliés par la flèche, nous voyons deux passages se correspondant à peu près, constituant un exemple clair de la mise en œuvre des espacements. Les différences entre le brouillon et le texte publié sont minimes. Le nombre de cercles ou carrés dans les brouillons indique exactement la longueur des espaces intralinéaires dans la version publiée. Il faut remarquer que si cela fonctionne généralement comme une science exacte, c'est parce que tous les caractères chinois occupent théoriquement le même espace sur la page, contrairement aux signes que l'auteur avait adoptés plus tôt ou contrairement aux unités linguistiques qui sont de longueurs diverses. La comparaison des manuscrits des deux romans montre que si le second conserve certaines des caractéristiques typographiques du premier, les blancs s'y déploient néanmoins jusqu'à y prédominer. Une des causes possibles de la convergence mentionnée plus haut pourrait être que comme ce protosystème ne devient jamais un véritable système (comme celui des notations musicales) ou ne fait pas partie intégrante du texte, comme dans les pièces de Beckett, la tâche démiurgique de création de ce protosystème est trop complexe pour être menée à son terme. Nous pourrions aussi supposer qu'en inscrivant dans le manuscrit cette tâche impossible, faisant ainsi du brouillon le champ de bataille où se joue la guerre des mots, le romancier, pour des raisons d'ordre pratique ou non, choisit de sculpter la page blanche, tentant de dompter le vide en le soumettant à l'exactitude de la mesure des blancs. Si dans le premier roman il commence par traiter le manuscrit comme s'il était le Texte ultime, dans le roman suivant c'est la page imprimée qui semble être devenue cette terra incognita de laquelle il s'efforce d'extraire une langue, une lingua franca, suffisamment précise pour son projet. Il y a une sorte d'hésitation dans la ponctuation, ainsi que dans la dynamique entre le manuscrit et le texte, qui fait de la limite entre le brouillon et le texte de Wang non pas une frontière bien définie mais plutôt une sorte de grève soumise à la fluctuation des marées. En observant les deux séries de manuscrits, il semble que la fabrication hésitante d'un système de signes soit visible, conservée dans les textes eux-mêmes, dans les expérimentations typographiques, les ponctuations idiosyncratiques, voire dans la façon de sculpter l'espace de la page. Dans le deuxième roman, on retrouve des traces de la genèse dans le tissu du texte parce que l'auteur a décidé, au cours du processus de développement de ses habitudes de composition, de simplifier les expérimentations typographiques et la ponctuation afin de les remplacer par un meilleur contrôle des blancs, et ces blancs ne font pas que réduire la différence entre le brouillon et le texte : la précision des blancs, qui permet un transfert exact du manuscrit au texte, les rapproche aussi l'un de l'autre en termes de statut. La distinction entre les deux statuts, «brouillon» et « texte », est ainsi appelée à être contestée, nous amenant à nous concentrer sur l'entredeux : il semble ainsi d'autant plus justifié de passer du « pré-texte » (Genette) à l'avant-texte, afin de mieux formuler la subtile négociation entre le manuscrit et le texte dans l'exemple de Wang.

En nous appuyant sur trois références très générales - deux articles de Pierre-Marc de Biasi et Jean Bellemin-Noël issus de Genetic Criticism et un article de Louis Hay publié dans un numéro spécial sur la critique génétique de Yale French Studies ${ }^{9}-$, nous pouvons envisager trois approches possibles de ces matériaux génétiques : une approche systématique, une approche analytique et une approche plus historique.

8. Je remercie le comité de lecture de Genesis pour m'avoir suggéré cette comparaison de la méthode de Wang avec la notation des pauses musicales et avec Beckett, ainsi que pour leurs suggestions et commentaires incisifs, ici et ailleurs.

9. J. Bellemin-Noël, « Psychoanalytic Reading and the Avant-texte » et P.-M. de Biasi, « Towards a Science of Literature: Manuscript Analysis », dans Genetic Criticism: Texts and Avant-textes, ed. Jed Deppman, Daniel Ferrer and Michael Groden, Philadelphia, University of Pennsylvania Press, 2004 ; L. Hay, « History or Genesis ? », Yale French Studies, n 89 , 1996, p. 192-207. 
Cette dernière consisterait, dans le prolongement de la présente étude, à comparer les brouillons obsessionnels de Wang à ceux d'écrivains contemporains en relation avec la présence ou l'absence de cette obsession. Ce travail devra faire appel à la psychanalyse dans la mesure où le processus d'écriture, pour Wang et certains de ses compatriotes, n'est qu'un aspect d'une vie passée à lire et écouter avec piété ou anxiété la voix des grands maîtres. De Biasi démontre avec précision que ce qui prouve la viabilité d'un dossier d'un point de vue génétique, c'est la possibilité de l'organiser, de le catégoriser, de le systématiser, enfin de l'éditer. Dans le cas de Wang, les aspects historiques et analytiques influencent l'aspect systématique, il est donc nécessaire de se pencher de plus près sur la façon dont ces deux tendances s'allient ou s'opposent.

\section{De quelques brouillons supplémentaires, tirés d'une œuvre en cours}

Nous nous appuierons ici sur l'étude d'une sélection de matériaux génétiques appartenant à une œuvre en cours de Wang Wen-hsing, dans le but d'explorer davantage les frontières instables entre le texte et ses avant-textes. La diversité de ces matériaux vient enrichir et compliquer notre problématique. Ils incluent en effet des fiches cartonnées, deux types de brouillons et des fragments de papier. Tout cela représente l'essentiel du dossier, auquel il faut ajouter des éléments non reproduits ici : une esquisse générale de la conception et de l'intrigue du roman et un canevas plus développé qui en découle. Ces deux éléments pourraient être considérés, avec les précautions qui s'imposent, comme le «scénario initial » et le "scénario détaillé10 ». Ces documents sont nécessairement sommaires et, comme nous le verrons plus loin, le véritable travail de composition repose sur la préparation des deux types de brouillons mentionnés plus haut, l'un que nous n'avons pas encore rencontré (par exemple fig. 10 et 11), l'autre qui nous est plus familier (fig. 12) : le travail de rédaction a lieu en particulier sur les fragments de papier (fig. 9 et 10). Ces trois types de documents forment un dossier unique qui donne un tour nouveau à ce qu'on appelle les esquisses.
Les documents présentés ici, mais aussi les propos de l'auteur, font apparaître que le travail préliminaire consiste à noter et à organiser des idées concernant le projet d'ensemble du roman à l'aide des scénarios et de fiches. Les fiches procurent en effet des informations qui vont de simples détails à de véritables décisions stratégiques. Elles remplissent plus d'une fonction et ne seront finalement pas toutes utilisées. Les deux fiches reproduites ici (fig. 7 et 8) traitent de sujets allant des noms des personnages à la question centrale de donner une forme romanesque au thème religieux.

Le travail de rédaction se situe simultanément dans les deux brouillons et dans les fragments qui servent de « traduction » pour le transfert d'un brouillon à un autre, faisant de ces trois documents une trinité, trois en un. Après le travail préparatoire des scénarios, la figure 11 illustre la phase qui précède la mise au net finale, mais l'exemple que nous voyons ici est lui-même le résultat d'un processus d'élimination et de remplacement d'une version par une autre mieux conçue. Ce processus de perfectionnement culmine au moment où Wang exécute une sorte de répétition générale du texte de la figure 11, en silence, phrase par phrase : en frappant sur la table, il cherche le rythme des mots, avant que le texte ne soit joué pour de bon, les figures 9 et 10 en montrant les grandes lignes de façon saisissante et la figure 12 enregistrant le résultat de la combinaison de ce qui se passe dans les figures 9 et 10 et du texte de la figure 11. Les rectangles des figures 9 et 10 indiquent des notes typiques. Chaque unité de scription correspond à une phrase ou à une expression du premier brouillon (fig. 11). Ces notes vont de tracés complètement illisibles à des caractères partiellement déchiffrables. Selon l'auteur, la présence de caractères lisibles ne signifie pas nécessairement que l'on approche de la version finale : autrement dit, le processus d'écriture ne suit pas nécessairement une progression linéaire de l'illisible vers l'émergence de caractères lisibles. Ceci peut s'expliquer par le fait que la plupart des

10. Ces termes sont issus du tableau de P.-M. de Biasi dans « Towards a Science of Literature: Manuscript Analysis » (art. cité, p. 53). La description des différents stades de la composition et des types de matériaux leur correspondant s'appuie sur une conversation récente avec Wang Wen-hsing. 

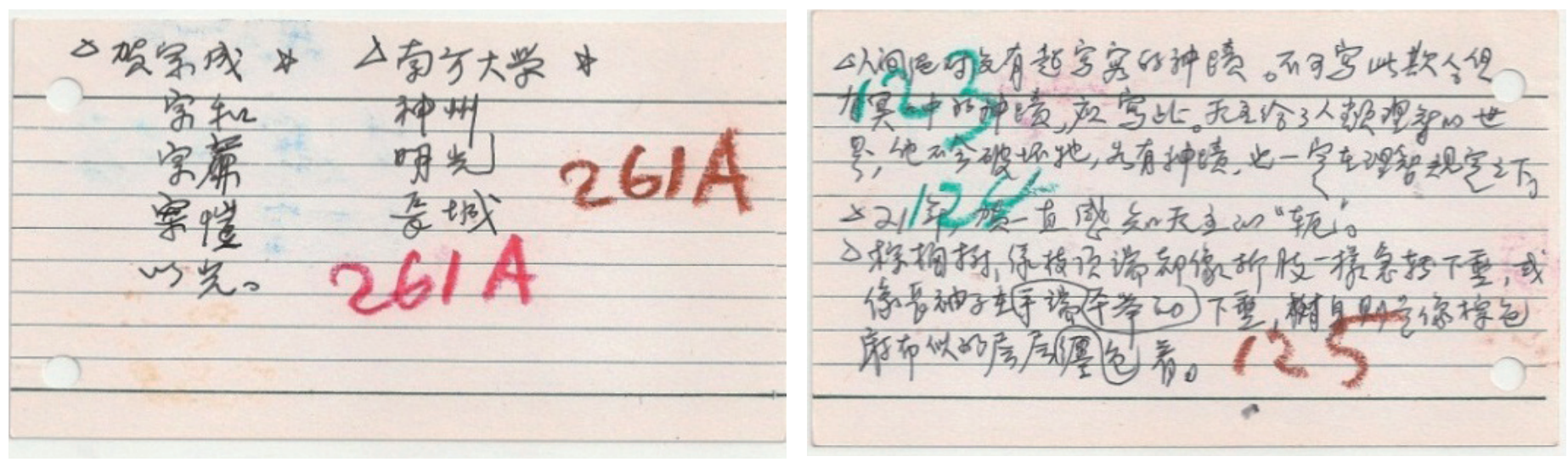

Fig. 7 et 8 : Wang Wen-hsing, fiches cartonnées d'une œuvre en cours (coll. privée)
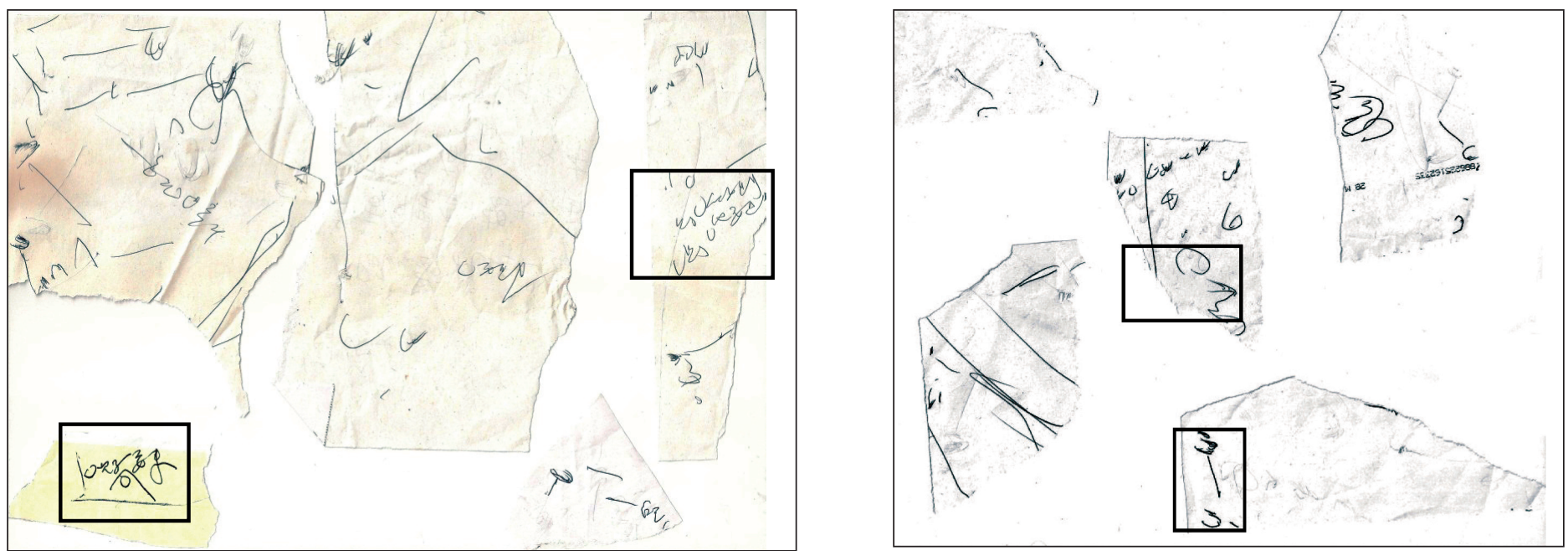

Fig. 9 et 10 : Wang Wen-hsing, fragments de papier pour une œuvre en cours (coll. privée)

mots sont en fait déjà fixés. Qu'ils apparaissent ou non n'altère pas la première décision et la difficulté ne réside pas dans le choix de ces mots.

Il faut généralement deux ou trois mois de travail pour « recopier » une seule page de brouillon du type que nous étudions ici (ou un collage de plusieurs petits fragments réunis pour former une «page »). Selon la chronologie établie par l'auteur lui-même, il a fallu six ans (de 1966 à 1972) pour achever Jiabian, un roman de deux cents pages environ dans la version de 1978. Le premier volume de Beihai de ren l'a occupé de 1974 à 1979 et le second de 1980 à 1997. Ainsi, il aura fallu vingt-trois ans pour publier les deux volumes, dont dix-sept pour le second. En moyenne, une année entière est consacrée à copier le dernier brouillon au net et une autre année pour la relecture. C'est pourquoi le premier volume, terminé en 1979 et long de cent quatre-vingt-deux pages, a été publié en 1981 tandis que le second, qui en fait cent quatre-vingt-cinq, a été publié en 1999, deux ans après l'achèvement du brouillon.

La production de ce type de brouillon se fait par vagues successives, c'est-à-dire que tous les brouillons ne sont pas prêts pour être transcrits tous en même temps. Ce n'est que lorsque toutes les phrases du brouillon existant ont été barrées (fig. 11) d'un ou deux traits horizontaux (par opposition aux barres obliques occasionnelles qui marquent l'endroit où une séance de recopie quotidienne se termine) et que toutes les phrases numérotées ont été «traduites» que le romancier entame un nouveau cycle de production de brouillons correspondant à un travail de copie de deux ou trois mois. 


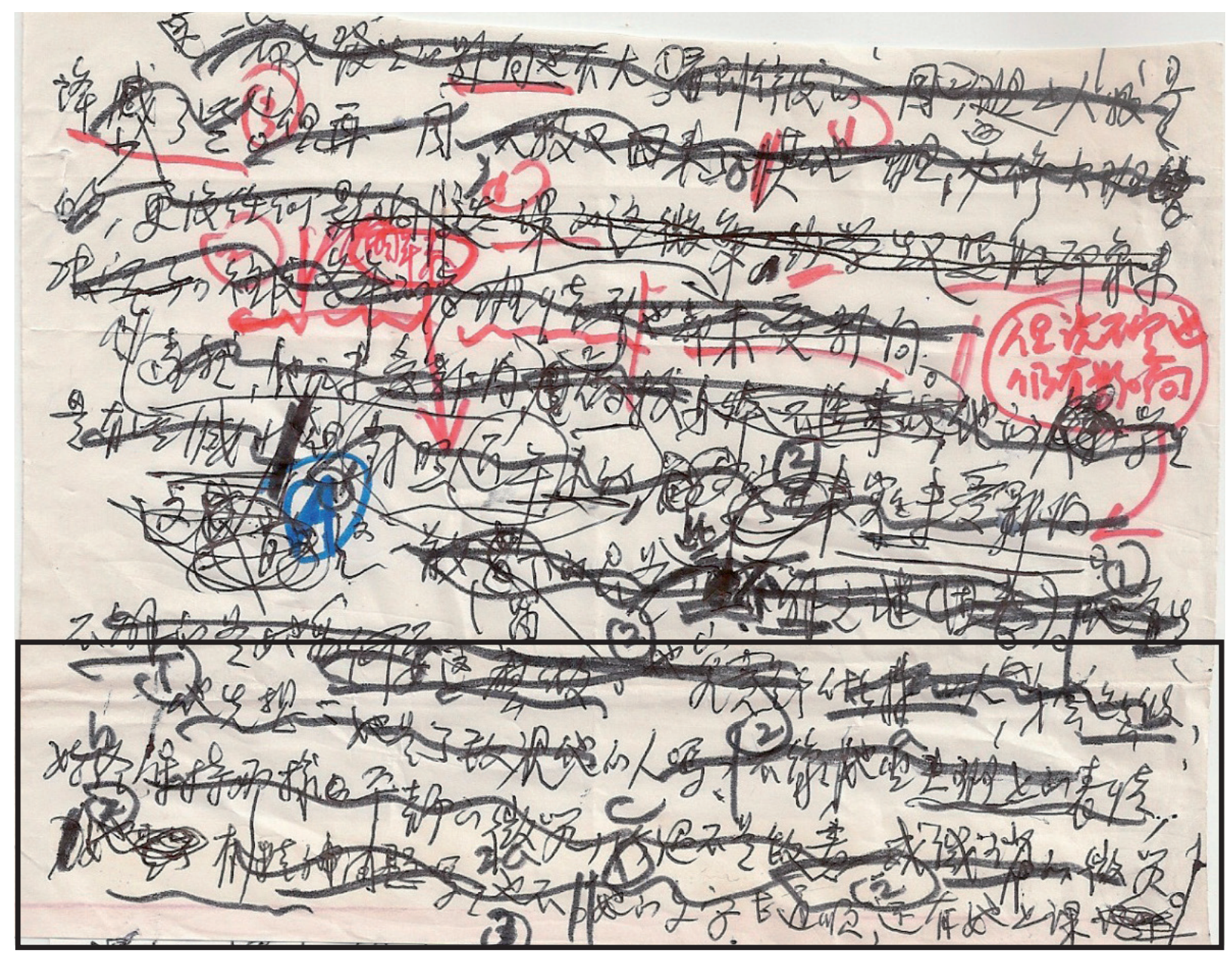

Fig. 11 : Wang Wen-hsing, brouillon d'une œuvre en cours (détail) (coll. privée)

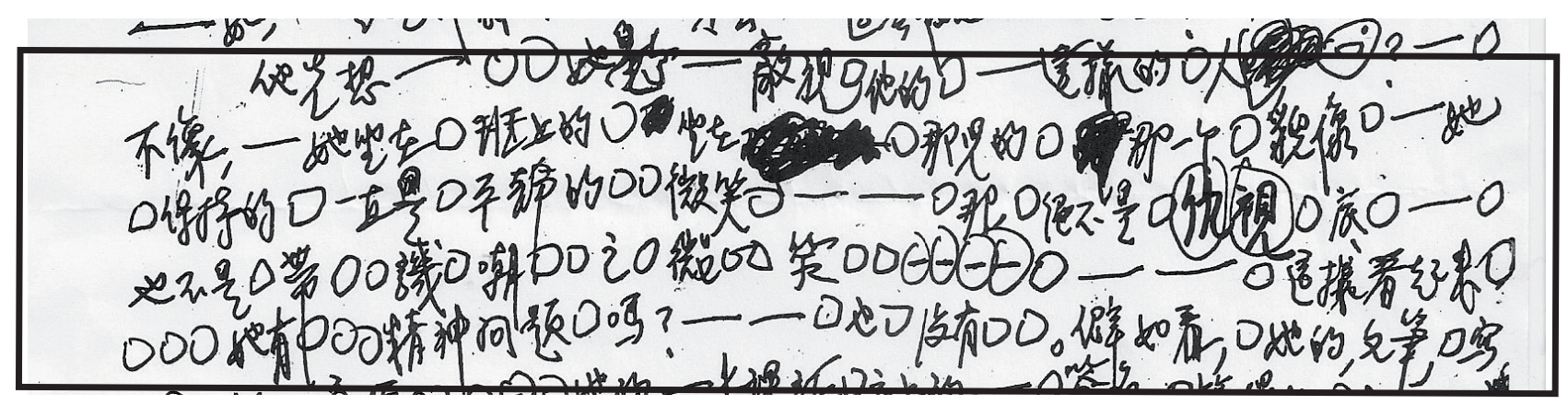

Fig. 12 : Wang Wen-hsing, brouillon d'une œuvre en cours, f. 50 r (détail) (coll. privée) 
Dans les figures 11 et 12 , nous avons encadré les phrases qui se correspondent à peu près. Nous appellerons la partie encadrée de la figure 11 « rouleau $\mathrm{A}$ » et celle de la figure 12 «rouleau B ». Nous n'en donnerons pour le moment qu'une traduction littérale, afin de mettre en évidence les concordances et divergences entre les deux. La transcription ci-dessous permet d'avoir une vue d'ensemble de tous les passages. Un tableau met ensuite l'accent sur le passage de A à B, par l'ajout de mots, l'insertion de signes de ponctuation, de mots vides ( $x u$ zi) et de blancs : $x u$ zi est une catégorie philologique ou grammaticale traditionnelle, qui s'oppose à celle des termes substantifs « réels » à laquelle appartiennent certains noms, verbes, adjectifs et quantifieurs. Les «mots vides » pourraient dénoter diverses catégories, allant des pronoms aux verbes modaux en passant par les interjections. Les mots vides qu'utilise Wang appartiennent principalement à cette dernière catégorie. Dans le brouillon étudié ici, nous avons 嗎, 的, 那兒的, 那個 et 之上的, qui, comme nous le verrons, correspondent à l'essentiel des modifications du rouleau B.

\section{Rouleau A (désormais A) :}

\section{(1) 他先想一她是個敵視他的人嗎? (2)不像 / 她坐在班上 的表情, \\ (1) Il se dit d'abord — Était-elle une personne qui lui était hostile ? (2) Peu probable / l'expression qu'elle avait assise en classe, \\ (1) 始終保持那樣X平靜的微笑, C那絕不是敵意或譏誚 的微笑。/ \\ (1) gardait toujours un tel $\mathrm{X}$ sourire calme, C Ce n'était absolument pas un rictus d'hostilité ou d'ironie. /}

(2) 她是個有精神問題嗎，2a 也不。//她的文字也通順， 還有她上課證上

(2) Avait-elle des problèmes mentaux. 2a Mais non. // Son écriture était plutôt fluide, et sur sa carte d'étudiante

\section{Rouleau B (désormais B) :}

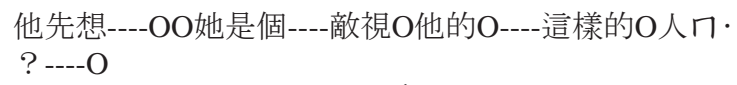

(1) Il se dit d'abord---- —OO Était-elle ainsi ----qui lui était hostile----une personne hum----O ?

不像，----她坐在 $\mathrm{O}$ 班上的 $\mathrm{O}$ 坐在XXX O那兒的 $\mathrm{O}$ X那一 個O貌像O----她

Peu probable, ----l'expression qu'elle avait assise $\mathrm{O}$ en classe $\mathrm{O}$ assise $X X X \mathrm{O}$ là [en classe] $\mathrm{O}$ X avec cette particulière $\mathrm{O}$ expression $\mathrm{O}----$ Elle

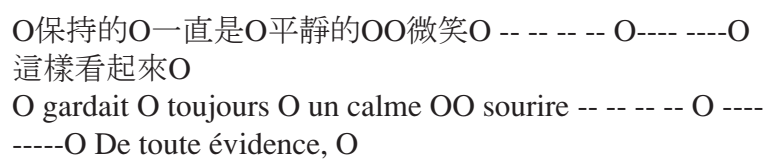

\begin{tabular}{|c|c|c|c|c|c|}
\hline A & $\begin{array}{l}\text { 他先想一她是 } \\
\text { 個敵視他的 } \\
\text { 人嗎* }\end{array}$ & $\begin{array}{l}\text { 不像 / 她坐在班上的 } \\
\text { 表情, }\end{array}$ & $\begin{array}{l}\text { 始蔠保持那 } \\
\text { 樣X平靜的微笑, C那 } \\
\square \text { 不是敵意或譏訬的微 } \\
\text { 笑。// }\end{array}$ & $\begin{array}{l}\text { 她是個有精神問題 } \\
\text { 嗎, 2a也不。// }\end{array}$ & $\begin{array}{l}\text { 她的文字也通 } \\
\text { 順，還有她上 } \\
\text { 課證上 }\end{array}$ \\
\hline B & $\begin{array}{l}\text { 他先想----OO } \\
\text { 她是個----敵 } \\
\text { 視O他的O---- } \\
\text { 這樣的O人 } \\
\text { П・*? ----O }\end{array}$ & $\begin{array}{l}\text { 不像, ----她坐在 } \mathrm{O} \text { 班 } \\
\text { 上的 } \mathrm{O} \text { 坐在XXX O那 } \\
\text { 兒的O X那一個O貌 } \\
\text { 像O--- }\end{array}$ & $\begin{array}{l}\text { 她O保持的O } \\
\text { 一直是O平靜的OO微笑 } \\
\mathrm{O} \text {-- -- -- O---- ----O }\end{array}$ & $\begin{array}{l}\text { 這樣看起來O } \\
\mathrm{OOO} \text { 她有OOO精 } \\
\text { 神問題O嗎? -- } \\
-----\mathrm{O} \text { 也 } \mathrm{O} \text { 沒有 } \\
\mathrm{OO}\end{array}$ & $\begin{array}{l}\text { 僻如看, } \mathrm{O} \text { 她 } \\
\text { 的文 } \\
\text { 筆, } \mathrm{O} \text { 寫得十 } \\
\text { 分 }<\mathrm{OO} \text { 通順 } \\
\mathrm{OOO}-\mathrm{O} \text { 她的 } \\
\text { 上課證 } \mathrm{O} \text { 之上 } \\
\text { 的 }\end{array}$ \\
\hline
\end{tabular}

* L'astérisque indique deux mots équivalents (嗎 et $\Pi \cdot$ ). Ils ont le même sens, mais le premier est un véritable caractère tandis que le second est représenté par le symbole phonétique correspondant. L'utilisation du gras et du soulignement dans le rouleau B indique les mots qui apparaissent pour la première fois. 
L'émergence du brouillon A remet-elle en question notre première interprétation des figures 2 à 5 (des éléments semblables au rouleau B) comme étant des brouillons ? Il semble que 1'on puisse considérer A et B comme l'aboutissement relativement tardif d'un long processus qui commence avec les scénarios. On pourrait même dire que B appartient davantage à la catégorie des mises au net, faisant de A le brouillon. L'auteur lui-même décrit la relation entre les deux documents en ces termes. La réponse à la question du statut de B (et par extension des brouillons des figures 2 et 4), comme étant une variété particulière de brouillon, une sorte de mise au net intermédiaire, dépend de la façon dont nous envisageons ce qui se passe entre les deux rouleaux : tandis que l'auteur envisage le processus comme une transcription, c'est-à-dire une copie, A et B sont conceptuellement les produits de différentes étapes, celles du brouillon et celle de la mise au net (intermédiaire). Selon cette conception, les signes qui prolifèrent dans $\mathrm{B}$ seraient alors véritablement des éléments « accidentels », soutenant mais ne décidant pas de la direction des éléments " substantifs », qui sont déjà en place au stade du brouillon.

Est-ce une pratique répandue que d'avoir une étape la mise au net intermédiaire, correspondant au rouleau B - qui soit à la fois proche de la fin du processus de rédaction, mais aussi d'un nouveau surgissement de complexité, c'est-à-dire d'une phase de remise en brouillon de la copie au net ? Pour répondre à cette question et pour voir dans quelle mesure il y a rupture ou continuité entre $\mathrm{A}$ et $\mathrm{B}$, nous devons rappeler les caractéristiques principales de Jiabian et de Beihai de ren : le semi-système de ponctuation, l'utilisation des blancs et la porosité de la frontière entre le texte et le manuscrit, autrement dit, l'approximation des deux. La juxtaposition des deux brouillons et la présentation en tableau permettent de faire apparaître le fait que les ronds (ou les blancs dans la version publiée), les tirets et les symboles phonétiques (c'est-à-dire le symbole $\Pi \cdot$, marqué par un astérisque, qui est habituellement équivalent au caractère 嗎) représentent l'essentiel du travail d'écriture, par et en eux-mêmes. Si, comme l'auteur et d'autres témoins l'ont affirmé, c'est là la partie la plus éprouvante, physiquement, de l'écriture, le travail de copie est en effet à la fois une épreuve physique et une tâche d'une nature radicalement différente. Le travail de transcription de $\mathrm{A}$ à $\mathrm{B}$ crée une distorsion au cœur de l'identité des deux documents : il s'agit bien de recopier, puisque les éléments « substantifs »sont fondamentalement identiques, avec une correspondance mot à mot le plus souvent, mais l'acte de transcription n'est pas qu'une répétition, puisqu'on assiste à une expansion, voire une explosion de la texture de l'œuvre, une texture évoquant la textualité des romans précédents, qui constitue l'identité du texte. La copie devient à la fois la tâche la plus concrète et la plus désincarnée puisque, lorsque l'auteur marque un temps au moment d'aboutir, l'écriture se répète à cause d'une nécessité pratique et en même temps se réaffirme par le biais des éléments « accidentels », des mots vides, des signes de ponctuation semi-systématiques et des calculs mesurant l'espace, qui tous sont des modifications ténues, immatérielles et difficiles à reproduire. Elles compliquent les dernières étapes de la genèse et ouvrent une dimension unique au sein de laquelle travaille l'auteur et où il tente tardivement de réinventer la langue dont il a hérité. Deux exemples d'une telle réinvention sont le symbole phonétique $(\Pi \cdot /$ 嗎 $)$ et les blancs, qui rejouent la répétition faussée entre $\mathrm{A}$ et $\mathrm{B}$. Bien que $\Pi$. et 嗎 soient théoriquement considérés comme identiques, l'auteur dote le premier d'une différence : une différence audible par des moyens graphiques. De même, les blancs entre les phrases (le contraste le plus marqué entre $\mathrm{A}$ et $\mathrm{B}$ ) rendent visible, presque palpable pour le lecteur, le passage du temps, donnant une dimension supplémentaire au corps du texte en le dotant de l'expressivité d'un accompagnement musical.

La nature particulière du travail de copie du brouillon (A) à la mise au net (B) permet d'une part de problématiser la spécificité de chaque document, d'autre part de rappeler que le travail de rédaction a lieu dans A, mais surtout dans B. Le statut complexe $\mathrm{du}$ brouillon relativement à la mise au net et le fait que la passion de l'écriture se ranime à cet instant, doit être rapproché du besoin de réinventer la langue, en creusant au burin la surface qui entoure les phrases, de manière à se concentrer sur l'intensité des vides qui précèdent la naissance du texte. Autrement dit, on pourrait s'appuyer sur le mode des pauses vides après l'écriture de A pour 
distinguer ce qui relève des conditions historiques d'une part et des implications systématiques du processus de combinaison et de sélection qui a lieu dans B d'autre part.

La précision extrême de cette procédure est particulièrement apparente dans le dernier groupe de documents que nous allons étudier. Les figures 9 et 10 sont des exemples des fragments de papier qui apparaissent entre A et B. L'une des raisons pour lesquelles l'auteur ou nous-mêmes pouvons considérer la transformation de A en B comme un processus de transcription ou de copie est que les deux séries de mots sont généralement identiques. C'est aussi parce qu'une comparaison entre A et B et l'étude détaillée des fragments montrent que la plupart des modifications ne portent pas sur des entités linguistiques : elles se concentrent sur des éléments suprasegmentaux et des griffonnages qui, ensemble, esquivent les mots euxmêmes. En fait, ce qui unit les brouillons et les fragments, c'est qu'ils traitent de la question de l'écriture en tant que telle très tardivement - tard, mais jamais trop tard, dans le processus génétique, juste avant la fin, indirectement, en empruntant une tangente par rapport au langage. Comme ces trois documents ne font qu'un, A ne peut pas plus être considéré comme un brouillon isolé que B ne peut être une simple mise au net.

Les figures 9 et 10 sont des exemples pris au hasard du stade le plus ardu entre A et B. Dans les deux exemples, cinq fragments sont réunis, reproduits ici ensemble. Les fragments sont de tailles et de formes variables, allant de quelques centimètres à un quart de feuille A4. Ils sont déchirés, sans méthode particulière, à partir de n'importe quel morceau de papier disponible. Chercher à réunir tous ces fragments ou à les trier pour y découvrir un ordre serait une tâche insurmontable. En moyenne, il y aurait vingt-cinq à cinquante fragments par séance de copie, correspondant à peu près à trente ou quarante caractères. Mais sur la plupart des fragments, l'espace est utilisé plus d'une fois pour noter plusieurs séries de tâtonnements et d'erreurs. Il y a parfois des inscriptions sur les deux côtés du papier, mais il est difficile de dire si le verso est indépendant du recto. Comme la plupart des fragments ne sont utilisés qu'une seule fois, il est peu probable que les fragments aient été utilisés à deux reprises indépendamment. Même en se limitant à l'équivalent d'un mois de travail, ce travail serait laborieux et écrasant, et la nature transitoire de ces documents les rend éphémères et pénibles à exploiter, quand ils n'ont pas été jetés après la « traduction » de A en B. S'il était possible de trier tous ces documents, on serait obligé de se demander ce que l'on ferait du résultat de ce tri, quel principe de sélection appliquer et dans quelle mesure les nouvelles technologies pourraient faciliter ce travail.

Les fragments portent essentiellement des griffonnages que seul l'auteur peut déchiffrer, et cette lisibilité même est de courte durée. Très rarement, quelques caractères émergent : la figure 9 rassemble des morceaux relativement lisibles, tandis que la plupart des signes de la figure 10 ne sont pas aisément déchiffrables. La première chose à faire pour étudier ces fragments est de revenir au lien entre A et B : l'objectif principal de ce travail patient de bricolage avec les mots et les blancs qui les entourent est de faire entendre une musique et une voix aussi précisément que possible, et comme il s'agit de prose, on ne s'étonnera pas de trouver des signes qui ressemblent à ceux utilisés pour noter les syllabes accentuées et non accentuées de la poésie anglaise (par exemple ' et ). Dans ces deux figures, en particulier dans les parties entourées d'un rectangle, le signe `remplace les mots afin de donner toute son importance à l'architecture d'ensemble, au sein de laquelle les mots ne jouent qu'un rôle partiel.

Trouver un moyen de conceptualiser le rôle des fragments nous amène aux limites de notre étude : l'écriture ou la rédaction caractérise le processus qui va des fiches aux scénarios, puis aux brouillons et à la mise au net, mais juste à la fin, les fragments représentent une forme alternative d'écriture/rédaction. On pourrait la considérer comme une forme de sténographie - où le signe ` représente des mots mais où, pour éviter de gêner l'intensité, voire la rigueur « scientifique » de la session, les caractères entiers sont réduits à ces quelques pattes de mouche. Mais pourquoi cette sténographie jouet-elle un rôle si indépendant et si décisif dans le processus d'écriture ? En outre, le travail de rédaction enregistré sur ces fragments fait partie intégrante de la dernière étape de l'écriture : une sorte de scansion a lieu entre A et B, par l'addition de blancs, de mots vides et de signes de ponctuation qui séparent et unissent les mots existants du rouleau $A$, qui fait émerger un rythme qui crée un contour d'ensemble pour le sens. Quelles conséquences cela a-t-il exactement sur le transfert de A à B ? Est-ce un processus de polissage, où l'auteur met la dernière main 
à une forme déjà en place ou un processus qui entraîne des transformations plus fondamentales déterminant la forme finale de l'œuvre, c'est-à-dire le sine qua non de la genèse ? Est-il même nécessaire de se pencher sur cette écriture sténographique, puisque le résultat resurgit de toute façon dans B ? C'est là bien sûr une des questions qui nous poussent à nous engager dans les études de genèse : qu'est-ce qu'un avant-texte, faut-il le définir et comment le définit-on par opposition au texte d'une part et au chaos de l'autre ? Je ne suis pas certain que la relation entre texte et avant-texte soit totalement analogue à celle entre les fragments et les deux brouillons. Le matériau demande peut-être un travail terminologique supplémentaire et exige que l'on aille plus loin dans la définition et la distinction des termes existants. Pour commencer, l'écriture opère à un niveau tellement infime, voire microscriptif 11 qu'il n'est peut-être pas substantiellement remarquable en termes d'étapes génétiques. Ainsi, la progression de la scansion est infime d'un point de vue génétique, mais décisive du point de vue de l'acte d'écriture en soi.

\section{Remarques de conclusion}

Nous avons commencé par le système de bricolage de Wang, cherchant constamment la clarté de l'expression par l'ajout infini de détails, ce qui nous a amené à tenter d'élaborer une taxinomie du brouillon et de la mise au net, en particulier en relation avec le déploiement des blancs, des mots vides et des éléments « accidentels », comme la ponctuation. Peut-être existeil une continuité plus profonde entre le premier protosystème de ponctuation et la mesure des espaces plus tardive. On peut même se demander s'il est possible d'envisager que d'autres semi-systèmes se dessinent. Cette ouverture caractéristique des brouillons se laisse percevoir dans les textes. Il est tentant de spéculer sur les motivations, internes et externes - si tant est qu'un terme de psychologie tel que «motivation » convienne ici - sous-jacentes à ces touches finales qui sont bien davantage que cela. Elles semblent en effet correspondre à une réouverture plutôt qu'à une clôture. Je crois que le défi lancé par une future exploration de ce travail de gravure microscriptif, semblable à une écriture cunéiforme artificielle, repose également sur la réouverture de ces forces opposées que sont une tendance vers une conclusion «naturelle » et le désir d'une deuxième chance et d'une deuxième vie, réunion de forces qui implique un travail au croisement de la recherche génétique et historique.

Traduit de l'anglais par Christine Collière

\section{Bibliographie}

WANG Wen-hsing

- Beihai de ren, 2 vol., Taïpei, Hong fen, 1999.

- Processus familial, trad. Camille Loivier, Arles, Actes Sud, 1999.

- Wang Wenxing shou gao ji : Jiabian, Beihai de ren (Les manuscrits de Wang Wenxing : Processus familial et Un homme dos à la mer), éd. Peng Yi, Taïpei, Flâneur, 2010.

11. J'ai dérivé le terme «microscriptif » du recueil Microscripts de Robert Walser (1878-1956), traduit de l'allemand (New York, New Directions and Christine Burgen, 2010). Dans son introduction, « Secrets, Not Codes: On Robert Walser's Microscripts », la traductrice, Susan Bernofsky, décrit l'intention de l'auteur comme une volonté de transformer l'acte humain et faillible de l'écriture en un processus « uniforme, mécanique », au moyen des microgrammes, des textes minuscules concentrés dans les plus petits recoins d'objets trouvés, fabriquant ainsi une « machine à écriture ». 
Peng Yi est professeur associé au département d'anglais de l'université centrale nationale de Zhongli (Taïwan). Sa thèse de doctorat porte sur Edmund Spenser et les théories de l'allégorie. Il a travaillé sur le penseur chinois Xun Zi, la ponctuation et la relation entre la psychanalyse et la pensée philosophique et politique fin-de-siècle en Chine, dans la perspective d'une réflexions sur les problèmes de la tradition et de la modernité. Il travaille actuellement sur les manuscrits chinois modernes et sur l'application des principes de la critique génétique aux manuscrits d'écrivains taïwanais contemporains, tels Wang Wen-hsing et Zhou Mengdie.

yipeng.yp@gmail.com

\section{Résumés \\ Marquer un temps, avant d'aboutir : les manuscrits de Wang Wen-hsing}

Cet article étudie les manuscrits du romancier contemporain taïwanais Wang Wen-hsing. À partir de deux romans publiés, dans lesquels la ponctuation et les espaces vides sont l'objet d'un travail continu, qui se poursuit tout au long des étapes de la rédaction, j'étudie dans cette perspective la transition d'un brouillon à l'autre et de l'esquisse à la mise au net. De l'un à l'autre, il n'y a pratiquement pas de différences à part la ponctuation, mais c'est là que se situe le véritable travail d'écriture. Le fait que le travail principal porte sur les suprasegmentaux nous renvoie aux premiers stades de l'écriture, mais aussi à deux manuscrits tardifs qui sont pratiquement identiques. Ils appartiennent à la phase la plus longue de la genèse qui précède immédiatement l'achèvement de la rédaction (le temps marqué avant l'aboutissement). En tenant compte de l'ensemble, j'espère saisir une évolution et montrer qu'elle aboutit à une esquisse et à une mise au net qui coïncident pratiquement dans le temps et par le contenu, mais dont la nature est tout à fait distincte. C'est la mise au net, ou le processus de copie d'un état à l'autre, qui constitue le cœur de l'écriture définissant les romans de Wang. La copie relance tardivement l'écriture, à la fin du processus de genèse.

This paper studies the manuscripts of a contemporary Taiwanese novelist, Wang Wenxing. Starting from two published novels where punctuation and empty spaces are constantly reworked, through all the writing stages, I will scrutinize this transition, from one draft to the other up to the fair copy. They are mostly identical except for the punctuation, but they constitute the labor of writing. That the main labor is on supersegmentals takes us back to early manuscripts but also forward to the two substantially identical later drafts; they represent the most time-consuming phase before the completion of the drafting process (i.e., the long pause before the end). By attending to these two, I hope both to trace an evolution and highlight how it culminates in a draft and a fair copy which are chronologically and substantially almost identical but in nature quite separate. The fair copy, or the process of copying from the earlier to the later, is at the heart of writing, defining Wang's novels. The copying out belatedly revives the writing, at the end of the genetic process.

Dieser Artikel betrachtet die Manuskripte des zeitgenössischen taiwanesischen Romanschriftstellers Wang Wen-hsing. Anhand zweier veröffentlichter Romane, in denen die Zeichensetzung und die Leerzeichen Gegenstand einer Arbeit sind, welche sich über die gesamten Phasen des Verfassens erstreckt, wird aus dieser Perspektive der Übergang von einem Entwurf zum nächsten und von der Skizze zur Reinschrift untersucht. Bis auf die Zeichensetzung gibt es praktisch keine Unterschiede zwischen den Texten. Aber genau dort findet die wirkliche Arbeit des Schreibens statt. Die Tatsache, dass sich die Haupttätigkeit auf die Suprasegmentalia konzentriert, verweist uns auf die ersten Stadien des Schreibens, aber gleichzeitig auch auf zwei späte, praktisch identische Manuskripte zurück. Sie tauchen in der längsten Phase der Genese auf, unmittelbar vor dem Abschluss des Textes (die lange Zeit vor dem fertigen Text). Durch die Betrachtung dieser Texte als Gesamtheit erhofft sich der vorliegende Artikel, eine Entwicklung zu erfassen und aufzuzeigen, dass diese in einem Entwurf und einer Reinschrift endet, welche zeitlich und inhaltlich praktisch übereinstimmen, deren Art jedoch gänzlich unterschiedlich ist. Es ist die Reinschrift, oder der Prozess der Kopie von einer Form zur nächsten, welcher das Herzstück des den Roman von Wang auszeichnenden Schreibens ausmacht. Das Abschreiben greift das Schreiben spät auf, am Ende des genetischen Prozesses.

El presente artículo estudia los manuscritos del novelista taiwanés contemporáneo Wang Wen-hsing. A partir de dos novelas publicadas (en las cuales la puntuación y los espacios en blanco son objeto de un trabajo permanente, que se prolonga a lo largo de las etapas de redacción), estudio en esta perspectiva la transición de un borrador a otro y de un esbozo a la copia en limpio. Entre unos y otros no existen casi diferencias, como no sea la puntuación, pero es precisamente allí donde se sitúa el verdadero trabajo de escritura. El hecho de que este trabajo principal esté dedicado a los elementos suprasegmentales nos remite a los primeros estadios de la escritura, pero también a dos manuscritos tardíos que son prácticamente idénticos. Corresponden a la fase más larga de la génesis, que precede inmediatamente la finalización de la redacción (el tiempo muerto antes del cierre final). Tomando en cuenta el conjunto, espero poder captar una evolución y mostrar que de ésta resultan un esbozo y una copia limpia que coinciden prácticamente en el tiempo y por su contenido, pero cuya naturaleza es totalmente distinta. Es la puesta en limpio, o el proceso de copia de un estado a otro, lo que constituye el corazón de la escritura que define las novelas de Wang. La copia reactiva tardíamente la escritura, al final del proceso de génesis. 
Este artigo estuda os manuscritos do romancista contemporâneo Wang Wen-hsing, de Taiwan. A partir de dois romances publicados, em que espaços em branco e pontuação são objecto de revisão contínua, em todas as fases da elaboração, estudo a transição de um rascunho para outro até à cópia limpa. De uns aos outros, não há praticamente diferenças além da pontuação, mas é nela que se situa o verdadeiro trabalho de escrita. Esta concentração sobre os supra-segmentais remete-nos para estágios iniciais da escrita, mas também para dois manuscritos tardios, que são praticamente idênticos. Pertencem à fase mais longa da génese, imediatamente anterior à conclusão da redacção. Julgo que se pode depreender disto uma evolução que leva a um esboço e a uma cópia limpa coincidentes no tempo e no conteúdo, mas de natureza totalmente distinta. É a passagem a limpo, a cópia de um estado ao outro, que forma o cerne da escrita definidora dos romances de Wang. A cópia revitaliza a escrita numa fase tardia, quando o processo genético se termina.

L'articolo esamina i manoscritti del romanziere taiwanese contemporaneo Wang Wen-hsing. Partendo da due romanzi editi, in cui la punteggiatura e gli spazi sono oggetto di un lavoro di ristrutturazione permanente, che occupa le diverse fasi redazionali, viene qui indagato il passaggio da un abbozzo all'altro, dalla prima traccia all'ultima revisione. Nel passaggio dall'uno all'altro stadio, si rilevano appunto le sole sostanziali differenze d'interpunzione; ed è proprio in quest'operazione che si rende evidente il reale lavorìo redazionale. Il fatto poi che il lavorìo prevalente si orienti su elementi soprasegmentali, induce naturalmente ad indagare sulle prime fasi di scrittura e sugli stadi redazionali tardivi tramandati da due manoscritti che, sostanzialmente, conservano la medesima lezione; appartenendo essi allo stadio il più lungo dell'itinerario genetico che precede la definizione del testo. Proprio considerando questa tradizione, si tenta di poter tracciare le linee di quella evoluzione e dimonstrando come esse si fondino su un progetto sia pur schizzato e su una "bella copia" che cronologicamente e sostanzialmente coincidendo, pur essendo tuttavia differenti nella loro natura. La "bella copia", ovvero l'itinerario di composizione (da uno stadio all'altro) che a quella "bella copia" conduce, costituisce l'elemento-chiave per intendere i romanzi di Wang. La copia riavvia tardivamente la scrittura alla fine del processo genetico. 